\title{
A Hybrid Approach for Artifacts Removal from EEG Recordings
}

\author{
Alaa Eldeen M. Helal \\ Dept of Biomedical \\ Engineering, Faculty of \\ Engineering, Helwan \\ University, Egypt
}

\author{
Ahmed Farag Seddik \\ Dept of Biomedical \\ Engineering, Faculty of \\ Engineering, Helwan \\ University, Egypt
}

\author{
Ayat Allah F. Hussein \\ Clinical Neurophysiology \\ Department, Faculty of \\ Medicine, Cairo University, \\ Cairo, Egypt
}

\begin{abstract}
The electroencephalogram (EEG) is a widely used traditional procedure for diagnosing, monitoring and managing neurological disorders. Many artifact types that often contaminate EEG remain a key challenge for precise diagnosis of brain dysfunctions and neurological disorders. Hence, artifact removal is intuitively required for accurate EEG analysis and treatment. This paper presents a new extensive method that can remove a wide variety of EEG artifacts based mainly on Template Matching approach including multiple signal-processing tools. The method was evaluated and validated on real EEG data, giving promising results that offer better capabilities to neurophysiologists in routine EEG examinations and diagnosis.
\end{abstract}

\section{Keywords}

Electroencephalogram (EEG), artifacts removal, independent component analysis, wavelet, cosine similarity measure

\section{INTRODUCTION}

Epilepsy is a common neurological disorder characterized by unprovoked recurring epileptic seizures. About $1 \%$ of people in the world suffer from epilepsy and $30 \%$ of them are not helped by medication [1]. Long-term monitoring of the electroencephalogram (EEG) is performed to identify abnormalities that perform hallmarks in neuronal disorders [2]. Investigation of Long-term EEG recordings is still done through neurophysiologists manually, by his/her naked eye. Visual examination of such massive EEG data is challenging as well as tiresome, very labor-intensive and too time consuming. Moreover, contamination with noise makes routine EEG examination is very problematic. The noises in the EEG are called artifacts that should be removed for proper analysis, diagnosis and treatment. These artifacts are generated from the body itself (bio artifacts) or caused by external interferences. Bio artifacts are originating from noncerebral sources as EMG, EOG and ECG but may be captured by an EEG machine. External artifacts are occurring during EEG recording due to bad electrodes, patient movements, baseline movement, electrical power line $(50 / 60 \mathrm{~Hz})$, mobile interference... etc [3]. The presence of such artifacts obscures the effective EEG waveforms to the extent that it may lead to wrong diagnosis with great danger of faulty drugs or inappropriate therapeutic protocols. Since the signals must therefore present a true and clear picture about brain activities, the importance of artifact removal comes to be very needful and urgent to overcome the difficulties of traditional EEG examination. Up to now, the most techniques have been proposed for removing artifacts can be classified into; Regression based method (AR) [4], Adaptive Filters [5], Principal Component Analysis (PCA) [6], Independent
Component Analysis (ICA) [7], and Wavelet Transform (WT) [8]. Although these presented methods are practiced, each of them not only was designed to remove one particular artifact with its own limitations, but ruefully also considerable information of the true EEG can be lost. A more detailed review of artifact removal techniques can be obtained from the literature in [9].

\section{PROPOSED APPROACH}

Indeed, from the signal processing point of view, the rejection of EEG artifacts can be regarded as a pattern recognition problem. So, despite more sophisticated approaches have been suggested, the main procedures (in a way or another) remain relatively unchanged and could be shortened in; preprocess the raw EEG to remove linear trends and signal drifts, digitize, segment and extract different characteristics (morphological, spectral, spatial, statistical, frequencytemporal), then these features are fed into a classification stage. In classification phase, the input feature vectors are analyzed according to a certain algorithm to identify artifact patterns along EEG signal. In the presented work, these main procedures are followed objectively to detect and remove 15 artifact types which are slow blink, fast blink, vertical eye movements, horizontal eye movements, slow eyes rolling, heartbeat (ECG), coughing, chair rocking, chewing, leg movements, neck movements (forward/backward), neck movements (left/right), shoulder movements (up/down), $50 / 60 \mathrm{~Hz}$ line noise and bad electrode artifacts. Our method presented in this paper is extensive so that; it recognizes various EEG artifacts, removes it by setting their components to zero, reconstruct the remaining pure signals and project them back into their original channels/electrodes. The new generalized method is based on analogizing (comparing) various features of the predefined artifact templates (derived and preserved in the system previously) against the same features extracted from a sequenced window of the raw EEG data under analysis. Our approach comprises two steps:-

(1) Derivation of template model for each artifact type and extract their significant features (as explained later in sections 3.2),

(2) Analogizing (comparing) various features of the predefined templates against the same features extracted from a sequenced window of the raw EEG data under analysis (as illustrated in sections 3.4).

A hybrid of processing tools was used to get characteristics embedded in time, space and frequency domains. Mainly, spectral, spatial and frequency-temporal features were analyzed to detect similar windows of the raw data to any artifact template. Lomb-Scargle Transform (LST) was used to study our method effect on spectral characteristics and overall 
quality of the cleaned signal after removing artifacts. Independent Component Analysis (ICA) and Symmlet Wavelet family (sym 8 ) are provided to extract features that will be analogized via a simple technique called "Cosine Similarity". Firstly, the Cosine Similarity measure is used as a pre-classifier in a manner that; if there are similarities (according to specified threshold for each template' feature) between the tested window and any template, the tested window is suspected to contain artifacts. This is illustrated by the block diagram shown in figure 1 . Secondly, to avoid deception (reducing the false detection rate) and true EEG data loss, a variable threshold determined by Mean Squared Error (MSE) is applied, to decide either the tested window is an artifact or a true EEG. Briefly, the tested EEG window will be considered an artifact, if and only if both outputs of Cosine Similarity measure and MSE are true (i.e. exceed their thresholds) else, it is a true EEG. These basic procedures in our algorithm will proceed sequentially; in very short windowing epochs to catch instant transients, overcome the rapid dynamics and nonlinear nature of EEG.

\section{MATERIALS and METHODS 3.1 Data acquisition}

Three real EEG data sets collected from Clinical Neuro Physiology Unit, Kasr El Aini Hospital, Faculty of Medicine, Cairo University. The data sets were investigated and examined by Professors of Clinical Neurophysiology Department. These data were categorized as:

\subsubsection{Dataset 1 (for deriving artifact templates)}

EEG segments for different artifact types were obtained during actual online EEG acquisition from 10 males and 10 females aged 18-57 years old. Artifacts were generated by requesting the participated subjects to do familiarized tasks intentionally or unintentionally, as eye blinking, eye rolling, teeth clenching, coughing, chewing, leg movements, neck movements, shoulder movements (up/down), ..... and swallowing. When the subject does a specified action for a time period, the corresponding recorded EEG was clearly marked by its belonging artifact label. Also, by the close examination and well investigation of many EEG recordings (mostly more than 400 hours) were previously stored in the Clinical Neuro Physiology Unit (Kasr El Aini Hospital), different 15 types of EEG artifacts were identified and labeled along its duration as $\left(\operatorname{art}_{1}, \operatorname{art}_{2}, \operatorname{art}_{3}, \ldots, \operatorname{art}_{15}\right)$. Several patterns for each artifact type were picked up and separated into discrete segments of 0.5-1 Sec. So, many artifact types were acquired, recorded and labeled using the True Scan EEG system (DEYMED Diagnostic, Czech Republic) which can import data into .mat format for further processing by MATLAB (Math-Works, R2012a). A template model for each artifact type is estimated based on these extracted segments (from the preserved recordings and EEG artifacts that acquired from the participated individuals online).

\subsubsection{Dataset 2 (for training and testing):}

This data set consisted of epileptic EEG patterns collected from 35 epileptic patients (whom had been under evaluation and treatment) and normal EEG data from 5 recent graduate physicians were trained in the Clinical Neurophysiology Unit. We concerned to include contaminated epileptic data in this tested set to quantify our algorithm performance on different epileptic waveform activities after artifact removal. Also, we interested to add normal EEG data to study our method effect on morphology, spectral, statistical and other characteristics of normal EEG signals.

\subsubsection{Dataset 3 (for power spectrum evaluation):}

This data set consisted of very pure (uncontaminated) 20 EEG signals from epileptic and normal individuals to test our algorithm performance, according to power spectrum analysis based on Lomb-Scargle Periodogram (LSP).

All real data sets were collected according to the standard 10/20 system from the EEG monitoring unit. The two data sets 2, 3 were optimally filtered to remove linear trends and signal drifts for improving the reliability of ICA decomposition. For this purpose, an analog anti-aliasing filter with sampling frequency $250 \mathrm{~Hz}$ and a band pass Butterworth filter with cutoff frequencies of $(0.1-100 \mathrm{~Hz})$ were used to attenuate EEG channel drift and better satisfy ICA's stationary assumption.

\subsection{Template derivation of artifact patterns}

Template matching is the process of detecting most prominent events in a signal by comparing it to a predefined template that characterized by certain features intimately pertaining to these events. In this study, to perform a certain template, appropriate segments corresponding to each artifact type were acquired online from many volunteers (whom requested to perform significant tasks to generate different artifact types) and other artifact patterns were picked up by qualified neurophysiologists from many EEG recordings (stored in the Neuro Physiology Unit, Kasr El Aini Hospital). The temporal, spectral and frequency characteristics of the extracted segments were analyzed to get precise, discrete artifact samples for each type. Based on these characteristics, some segments were discarded and the convenient remaining ones were indexed in separate patterns (mostly, 140-150 segments with duration from 0.5 to $1 \mathrm{Sec}$ for each). The proper patterns were categorized as $\left(\operatorname{art}_{1}, \operatorname{art}_{2}, \operatorname{art}_{3}, \ldots, \operatorname{art}_{15}\right)$ and selected for template derivation. The template for each artifact type $\left(\mathrm{tmp}_{1}\right.$, $\mathrm{tmp}_{2}, \mathrm{tmp}_{3}, \ldots, \mathrm{tmp}_{15}$ ) was estimated as the median of its corresponding segments. The median was used instead of average because it is more robust to outliers and signal trends.

\subsection{Feature extraction}

\subsubsection{Independent Component Analysis (ICA) and Wavelet Transform (WT)}

Independent Component Analysis (ICA) is a statistical technique by which mixtures of observed random data are linearly separated into maximally independent components from each other. It is the most recent and widely mathematical tool used of Blind Signal Separation (BSS) approaches.

Let $\mathbf{x}(\mathrm{t})$ denote a vector of $n$ observed signals $\mathrm{x}$ at time $t$;

$\mathbf{x}(t)=\left[x_{1}(t), x_{2}(t), \ldots, x_{n}(t)\right]^{\mathrm{T}}, \mathrm{x} \in \mathcal{R}^{n}$

where, $\mathbf{x}(t)$ is a mixture of $m$ unknown sources (s);

$\mathbf{s}=\left[s_{1}, s_{2}, \ldots, s_{m}\right]^{\mathrm{T}}, \mathbf{s} \in \mathcal{R}^{m}$

We target to separate the sources that compose the EEG signal. These sources are hypothesized to be a mixture of neural cortex sources (true EEG) and other interferences that cause contamination of the EEG signal. If we able to separate such interfered (artifacts), we can remove them and succeed to purify the EEG signal. The aim of using ICA is to do so by estimating the separating (unmixing) matrix $\mathrm{W}$, to obtain independent components $\mathrm{Y}$, such that;

$\mathrm{Y}=\mathrm{Wx}$

Eq. (3) implies that; 


$$
\mathrm{X}=\mathrm{W}^{-1} \mathrm{Y}
$$

Onton, et al., in [10] has shown that; the resulting $\left(\mathrm{X}_{i}=\mathrm{W}_{i}^{-1}\right.$ $\left.\mathrm{Y}_{i}\right)$, forms the spatial independent components $\left(\mathrm{X}_{i}\right)$, and the whole data $(\mathrm{X})$ are the sum of these ICs $\left(\mathrm{X}_{i}\right)$;

$$
\mathbf{X}=\sum \mathrm{X}_{i,} \quad \text { where, } i=1,2,3, \ldots \ldots n
$$

Thus, the inverse matrix $\left(\mathrm{W}^{-1}\right)$ contains relative weights (spatial features) that denote components' source locations on the scalp topography [11]. Several studies have also used ICA technique for EEG artifact removal [12-14]. Consequently, in our method, it was suggested that; by comparing the spatial features (embedded in the inverse matrix $\mathrm{W}^{-1}$ ) for both sequenced windows (segments of the signal under analysis) and each artifact template (according to an appropriate threshold), waveforms that resemble any of artifact templates $\left(\mathrm{tmp}_{1}, \mathrm{tmp}_{2}, \mathrm{tmp}_{3}, \ldots, \mathrm{tmp}_{15}\right)$ along the tested EEG signal would be recognized. Hyvärinen in [15] proposed a fast algorithm called FastICA to separate independent components in non Gaussian distribution signals (since the EEG signal contaminated by many artifact types has a nongaussian nature, FastICA has been very appropriate for our application). Also, FastICA has many significant advantages, such as faster convergence speed of iteration and better performance of noise immunity. At the same time, despite ICA has been vastly employed in many methods to reject artifacts from EEG, its capabilities to detect some artifact types are limited, especially when these artifacts overlap with the true EEG [16, 17]. Also, its performance depends on the size of the data set and cannot filter the artifacts without discarding the true signals as well, resulting in some effective data loss [18]. To overcome these limitations, the performance can be improved by extracting more features in other spaces beside that obtained by ICA. Considering the nonstationary nature of EEG signals (i.e. the frequency spectrum varies through time) [19], WT capability to transform the signal into time and frequency domains according to desired resolutions gives a superior macroscopic vision to extract features at different time localizations [20]. Therefore WT can separate any overlapping artifacts with the true EEG signals that ICA cannot filter out by distinguishing their temporal- frequency features [18]. Thus, like ICA, Wavelet Transform (WT) has been widely used in EEG denoising [21, 22] as well as other biomedical signal processing. However, using Wavelet Transform (WT) alone in EEG denoising cannot completely reject all artifacts, particularly when including comparable amplitudes with the true EEG signals and it may result in serious problems such as the Gibbs effect which distort the data $[18,23]$. Recently, researchers advised to combine Wavelet Transform (WT) and ICA to take advantages of both and exploit their benefits that complement each other [18, 23] where;

(i) WT removes overlapped artifacts that ICA can't filter out, especially in small data sets.

(ii) ICA can separate artifacts with the same amplitudes or higher than true EEG signals while WT has difficultly to do so.

Compressed versions of Wavelet functions match the highfrequency components, while stretched versions match the low-frequency components. For a conventional mother wavelet $\psi(t)$, the Discrete Wavelet Transform (DWT) at scale " $a$ " and position " $b$ ", is defined as;

$$
\Psi_{(a, b)}(t)=2^{a / 2} \psi\left(2^{a} t-b\right)
$$

Where, $a$ and $b$ are discrete values based on powers of two, $\left\{a_{j}=2^{-j}, b_{j, k}=\mathrm{k} 2^{-j}\right\}, j$ and $k$ are integers, the function $\Psi_{j, k}(t)$ is determined as;

$$
\Psi_{(j, k)}(t)=2^{-j / 2} \psi\left(2^{-j} \cdot t-k\right)
$$

By varying " $b$ ", the wavelet function $\psi_{a, b}(t)$ is replaced into the desired point of time, while capturing the high and lowfrequency components of the signal as increasing and decreasing " $a$ " respectively. So, at different locations of time, the frequency details of the signal can be obtained at several scales. With convenient wavelet, by sequential scaling and translating (varying parameters " $a$ " and " $b$ "), the signal is decomposed into sub-bands (WT coefficients), revealing the time- frequency features of the signal at different locations. Hence, both frequency and time features of fast transients in the EEG signal can be extracted from its WT coefficients, ignoring the nonstationary nature of the signal [24]. So, by correlating the EEG signal with wavelet functions of different sizes, the details of the signal can be obtained at several scales. Therefore artifacts would be detected by using an appropriate wavelet function and specific scales corresponding to the frequency bands in which these artifacts occupied. Signal decomposition by WT is performed by many Wavelet functions, called "mother" wavelet (the basis function $\psi$ ). For DWT, many wavelet families are available as Haar, Daubechies, Symlets, Coiflets, ..., etc. The quality of the features extracted by WT is mainly depends on the choice of a convenient "mother" wavelet and the number of decomposed sub-bands (WT coefficients) [24]. Selection of mother wavelet and number of WT coefficients is essential to obtain meaningful features retained in the wavelet coefficients at specific frequency ranges. Therefore, the chosen mother wavelet and number of decomposition levels (sub-bands) are subjective for each application, and yield to several practical experiments to satisfy the best results. In the presented method, we used Symmlet family of Wavelets due to its orthogonal and regularity as increasing the number of moments [25]. Also, this type of Wavelet family (Symmlets) was chosen since it gives a fast access to both WT and its inverse (IWT) on the basis of multi-resolution analysis, providing a very convenient way for further processing by Cosine Similarity measure. Practical experiments confirmed the best number of vanishing moments was 8 (Sym8) with 5level decomposition of the approximate and detail components. Hence, either of the tested EEG windows (segments of raw EEG data under analysis) and each artifact template $\left(\mathrm{tmp}_{1}, \mathrm{tmp}_{2}, \mathrm{tmp}_{3}, \ldots, \mathrm{tmp}_{15}\right)$ were decomposed into 6 sub-bands: 5 details (D1-D5) and one approximation (A5) to obtain the desired frequency resolution as explained in Table 1

Table 1: Frequency sub-bands corresponding to five-levels of DWT decomposition.

\begin{tabular}{|l|c|c|}
\hline Sub-bands & Frequency range (Hz) & Decomposition level \\
\hline D1 & $62.5-125$ & 1 \\
\hline D2 & $31.25-62.5$ & 2 \\
\hline D3 & $15.6-31.25$ & 3 \\
\hline D4 & $7.8-15.6$ & 4 \\
\hline D5 & $3.9-7.8$ & 5 \\
\hline A5 & $0-3.9$ & 6 \\
\hline
\end{tabular}

On feature extraction, statistical analysis offer better tools to extract significant features, providing a compact representation of the signal with a low computational 
complexity. So the maximum, minimum, mean, and standard deviation for each of the decomposed levels (D1-D5 and A5) were computed and set out as the feature vectors. Furthermore, the line length was used as another feature, since it is sensitive to amplitude-frequency variations [26] and its low computational time. In current work, for each artifact template $\left(\mathrm{tmp}_{1}, \mathrm{tmp}_{2}, \mathrm{tmp}_{3}, \ldots, \mathrm{tmp}_{15}\right)$, the spatial, wavelet (statistical and line length) features were extracted and ranked as $\mathrm{N}$ rows of an $\mathrm{N} \times \mathrm{M}$ matrix, then it is reshaped into an $\mathrm{N} \cdot \mathrm{M}$ size vector, (since the length is not the same in all feature types). Thus, a specific vector feature for each artifact template involves adequate attributes that depict its distribution over the features-plane space. Accordingly, identical procedures will be performed for each consecutive window of raw EEG data under test, to compose its vector of features.

\subsection{Cosine Similarity measure}

The cosine function is one to one, because it has only a fixed one output (neatly bounded between -1 to 1 ) specified for each tiny input. This significant property can be particularly employed to detect a resemblance (coincidence) between two patterns (in other words, to what extent two or more samples are similar or different to each other), by measuring the relative cosine value between their vectors of features at different localizations. Using the cosine function for such purposes is called Cosine Similarity measure. Compared to other tools, simplicity, accuracy and higher resolution give it many preferences to be utilized for pattern recognition and other signal processing tasks. Currently, this technique is widely implemented in many applications such as fingerprints, text and spectrum classification, signature investigation, speech and face recognition. By using this technique, similarity between patterns is determined according to the vectors' directions, not on the actual locations (as Euclidean Distance method) of the data, avoiding more complex computations and making this technique appropriate for fast transients and chaotic nature of EEG. This is detailed in [27], where this approach was used to measure similarity between two patterns (without requiring a priori knowledge of the tested data or a training stage). Powered with good results in [27] the author recommended applying this technique for audio recognition and detecting variability of beat to beat in ECG signals. Furthermore, this measure is used, in [28] to purify the tested EEG signal from EOG artifacts. By taking waveforms from the EOG channel as artifact patterns and reject the similar morphologies in the other EEG channels. In the presented work, the cosine similarity was provided to detect similar waveforms to any artifact template $\left(\mathrm{tmp}_{1}, \mathrm{tmp}_{2}, \mathrm{tmp}_{3}, \ldots, \mathrm{tmp}_{15}\right)$ along the tested EEG data (according to a specific threshold for each artifact type). Suppose that;

$$
\boldsymbol{Y}_{\boldsymbol{i}}=\left[\boldsymbol{y}_{1}, \boldsymbol{y}_{2}, \boldsymbol{y}_{3}, \ldots \ldots, \boldsymbol{y}_{M}\right]
$$

is the vector feature of the $i$-th window (of the raw EEG data under analysis), and

$$
X_{j}=\left[x_{1}, x_{2}, x_{3}, \ldots \ldots \ldots, x_{M}\right]
$$

is the vector feature of artifact templates $\left(\mathrm{tmp}_{1}, \mathrm{tmp}_{2}, \mathrm{tmp}_{3}, \ldots\right.$, tmp $_{15}$ ), for $j=1,2, ., \ldots, 15$ respectively. The cosine value of the angle $\boldsymbol{\theta}_{i j}$ between the vector features of $i$-th window $\boldsymbol{Y}_{\boldsymbol{i}}$, and $\boldsymbol{j}$-th template $\boldsymbol{X}_{\boldsymbol{j}}$ is;

$$
\cos \theta_{i j}=\frac{\sum_{q=1}^{M} Y_{i q} X_{j q}}{\sqrt{\sum_{q=1}^{M} Y_{i q}^{2} \sum_{q=1}^{M} X_{j q}^{2}}}
$$

To avoid the periodic nature of the cosine function, the absolute value of $\cos \boldsymbol{\theta}$, is determined to assess the extent of similarity between the $i$-th window and $j$-th template. Equation (10) implies that; the larger value represents more similarity (meaning the angle $\boldsymbol{\theta}$ is close to $\mathbf{0}^{\mathbf{0}}$, (i.e. both vector features, $\boldsymbol{Y}$ and $\boldsymbol{X}$ are nearly coincided and matched to each other). Practically, an appropriate threshold, $\boldsymbol{T}_{\boldsymbol{j}}=\left|\boldsymbol{\operatorname { c o s }} \boldsymbol{\theta}_{\boldsymbol{j}}\right|$, was adopted to each template, where $\boldsymbol{\theta}_{j}$, is the angle at which similarity is ascertained. So, similar waveforms to artifact templates $\left(\mathrm{tmp}_{1}, \mathrm{tmp}_{2}, \mathrm{tmp}_{3}, \ldots, \mathrm{tmp}_{15}\right)$ will be identified when; $\left(\left|\boldsymbol{c o s} \boldsymbol{\theta}_{i j}\right|>\boldsymbol{T}_{j}\right)$, for $j=1,2,3, \ldots, 15$ respectively. On the other hand, an adaptive model of Mean Squared Error (MSE) was used to avoid false detections of true EEG that may resemble artifact templates. MSE is the expected value of the square error by which the $i$-th window $\mathrm{Y}_{\mathrm{i}}$ differs from the $j$-th template $\mathrm{X}_{j}$, as;

$$
M S E_{i j}=\left[\mathbf{Y}_{i}-\mathbf{X}_{j}\right]^{2}
$$

The $i$-th window $\boldsymbol{Y}_{\boldsymbol{i}}$, will be considered an artifact when the following condition is satisfied;

$$
\mathrm{MSE}_{\mathrm{ij}}=\left[\mathbf{Y}_{\mathrm{i}}-\mathbf{X}_{\mathrm{j}}\right]^{2}>\gamma_{\mathrm{j}}
$$

Where $\gamma_{j}$, is a predefined threshold corresponding to each artifact template $\left(\mathrm{tmp}_{1}, \mathrm{tmp}_{2}, \mathrm{tmp}_{3}, \ldots, \mathrm{tmp}_{15}\right.$ for $j=1,2$, $3, \ldots, 15$ respectively). It should be noted that, as schematically shown in figure 2, the tested EEG window will be considered an artifact $\left(\operatorname{art}_{1}, \operatorname{art}_{2}, \operatorname{art}_{3}, \ldots, \operatorname{art}_{15}\right)$, if both outputs of Cosine Similarity measure and MSE are true (i.e. exceed their thresholds) else, it is a true EEG.

\subsection{Experimental procedures}

To get a clear and very precise template for each artifact type, two procedures were performed;

- First, 10 males and 10 females aged 18-57 years old were participated in actual EEG acquisition under supervision of EEG experts, utilizing the True Scan EEG system (DEYMED Diagnostic, Czech Republic). Artifacts were generated by requesting each subject to do significant tasks as eye blinking, eye rolling, teeth clenching, swallowing... etc. So, along the recoded EEG of the 20 subjects, corresponding contaminated EEG segments were marked along its duration and labeled according to its artifact type.

- Second, by the close examination and well investigation of many EEG recordings were stored in the Clinical Neuro Physiology Unit, different 15 types of EEG artifacts were identified and labeled as $\left(\operatorname{art}_{1}, \operatorname{art}_{2}, \operatorname{art}_{3}, \ldots, \operatorname{art}_{15}\right)$.

By focusing on the frequency-temporal and spectral characteristics of different artifacts, several patterns for each type were picked up from the two procedures and separated into discrete segments (mostly, 140-150 segments with duration from 0.5 to $1 \mathrm{Sec}$ for each). A template model for each artifact type is estimated based on the median of these extracted segments. The artifact templates were indexed as $\left(\mathrm{tmp}_{1}, \mathrm{tmp}_{2}, \mathrm{tmp}_{3}, \ldots, \mathrm{tmp}_{15}\right)$, and exported into binary format for further processing in MATLAB. To determine the cosine similarity and MSE thresholds $\cos \boldsymbol{\theta}_{j}$ and $\gamma_{j}$, after several experiments, it was found that, each artifact type has its own thresholds. Consequently, each artifact template is combined with its threshold as $\left\{\left(\mathrm{tmp}_{1}, \cos \theta_{1}, \gamma_{1}\right),\left(\mathrm{tmp}_{2}, \cos \theta_{2}, \gamma_{2}\right)\right.$, $\left.\left(\mathrm{tmp}_{3}, \cos \theta_{3}, \gamma_{3}\right), \ldots . .,\left(\operatorname{tmp}_{15}, \cos \theta_{15}, \gamma_{15}\right)\right\}$, and each of such combination was taken into account in designing our algorithm. 


\section{RESULTS}

The proposed method was implemented using Matlab R2012a (7.14.0.739) on a $2.10 \mathrm{GHz}$, Intel Core i3 laptop. To test our method, it was applied to the data set 2 , which acquired from 35 epileptic patients (20 males, 15 females) and normal EEG data from 5 healthy subjects aged 14-65 years. We concerned to include both epileptic and normal data in this tested set to investigate the effect of our algorithm on the morphology of epileptic waveforms and study the signal quality of normal EEG after artifact removal. Very bad 20 EEG signals contaminated with different artifact types and other uncontaminated (pure) 20 EEG ones (data set 3) were selected by Professors in Clinical Neurophysiology. The performance has been evaluated according to the conventional examination by experienced neurophysiologists where their decision was used as the "gold standard". In detail, during the visual investigation of the tested data, neurophysiologists were asked to mark each contaminated epoch, by labeling the artifact type and its duration. Labeling of different artifact types was done prior to applying our algorithm. After applying our method, neurophysiologists were then instructed to list any epochs of the tested data which they believe it was still contaminated. Later, the performance of the method was assessed based on the report made by the experts. Fig. 3 shows a set of contaminated EEG signals before and after our method is applied.

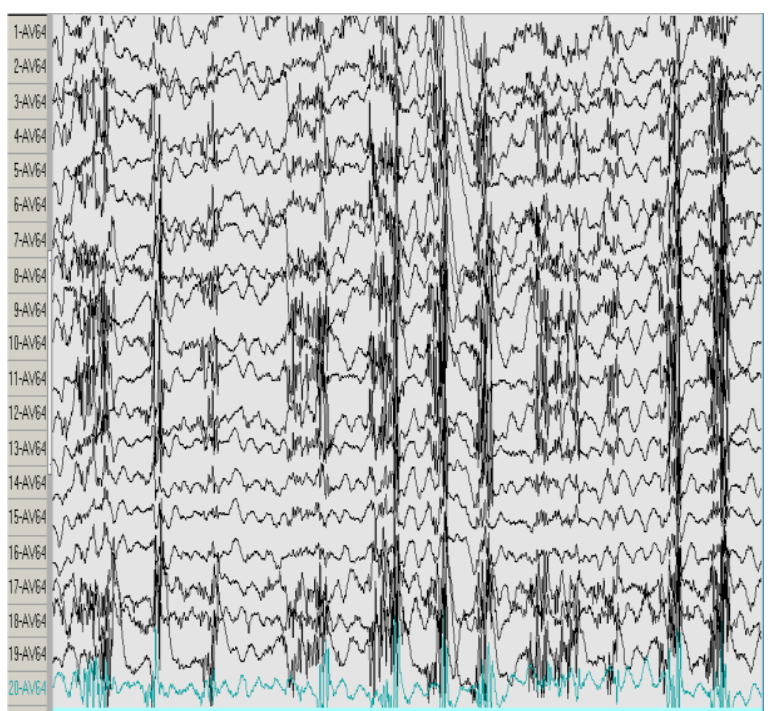

(A) Sample of raw contaminated EEG Signals

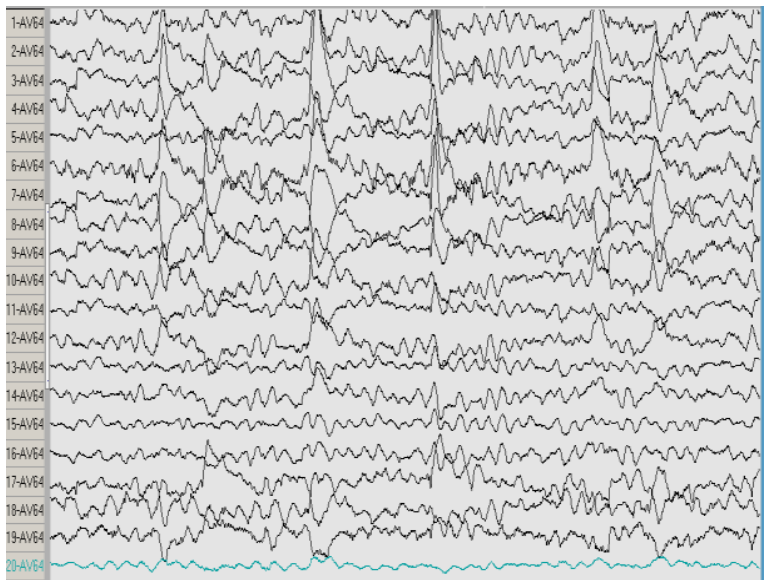

(B) EEG Signals after denoised with our algorithm

Fig 3: Contaminated EEG signals before (A) and after denoising (B)
Fig.3. A, shows one mixed EEG data set where overlays and different artifact types are existed in the most 20 signals. Figure 3.B, shows the same signals after applying our denoising method, indicating the overlays have been minimized and most artifacts were removed. Moreover, the cleaned signals are highlighted as its morphology (spikes, sharp waves, low frequency amplitudes, complexities ...etc.) has been more prominent than its originals (before the method is applied). This last observation is wonderful and very important since it illustrates that the characteristics of the true EEG signal weren't only changed but also both its signal to noise ratio (SNR) and the whole signal quality have been improved. However, more improvements could be gained via iterating the method by applying it to the same contaminated signals and repeated many times to refine the results and obtain the most overall performance (without distortion of the true EEG signals). This significant advantage can be shown in Fig. $3 \mathrm{~B}$, where some EOG artifacts have not been removed well in signals $2-8$, while after repeating the method one time again, these artifacts were reduced and the signal quality were getting better as shown in Fig. 4.

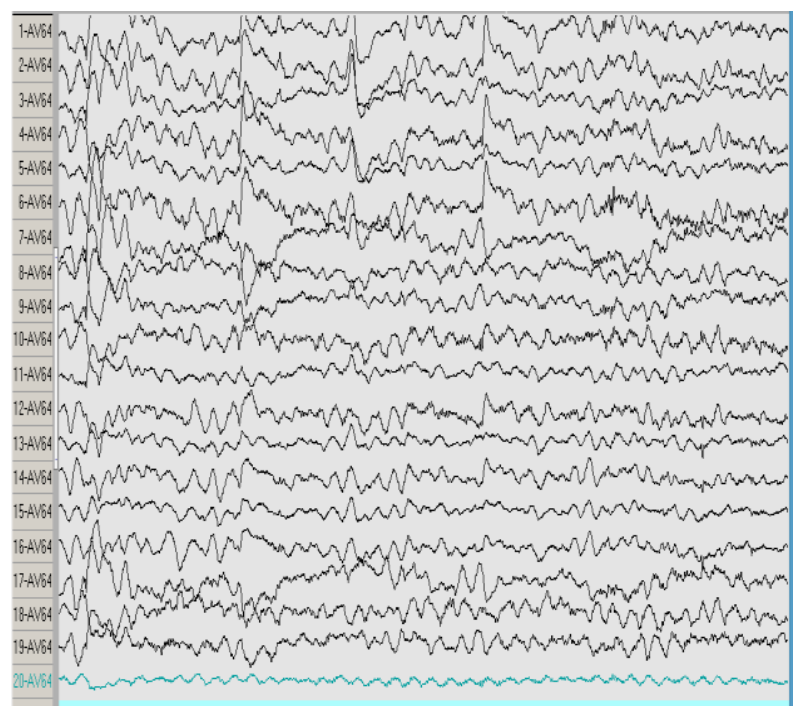

Fig 4: Refinement of very bad signals by iterating the method

Also, the performance was evaluated according to metrics of sensitivity and specificity. The first, sensitivity, was the percentage of true artifacts that were detected by the method to the total number that marked by neurophysiologists. The second, specificity, is the percentage of artifact-free EEG segments that were wrongly classified as contaminated to the total number of uncontaminated segments. Table 2 summarizes percentage of the two measures for each artifact type in the tested data. The measures of both Sensitivity and Specificity are formalized as;

Sensitivity $=($ detected artifacts $/$ total contaminated segments marked by the experts) $\times 100 \%$

\section{Specificity $=\left[1^{-}(\right.$detected false artifacts/uncontaminated} segments)] $\times 100 \%$ 
Table 2 Sensitivity and Specificity for each artifact type in the tested EEG data.

\begin{tabular}{|l|c|c|}
\hline Artifact type & SEN\% & SPEC\% \\
\hline Slow blink & 94.47 & 91.29 \\
\hline Fast blink & 95.59 & 81.83 \\
\hline Vertical eye movements & 89.04 & 90.88 \\
\hline Horizontal eye movements & 92.01 & 88.86 \\
\hline Slow eyes rolling & 78.34 & 93.58 \\
\hline Heart beat & 97.16 & 78.37 \\
\hline Coughing & 98.42 & 90.15 \\
\hline Lean chair rocking & 79.03 & 90.12 \\
\hline Chewing & 85.94 & 94.28 \\
\hline Legs movements & 68.17 & 96.07 \\
\hline Neck movements (forward/backward) & 90.14 & 83.13 \\
\hline Neck movements (left/right) & 96.01 & 86.41 \\
\hline Shoulders movements (up/down) & 83.95 & 91.80 \\
\hline 50/60Hz line noise & 97.19 & 91.47 \\
\hline Bad electrode noise & 96.11 & 73.19 \\
\hline Total & $\mathbf{8 9 . 4 3 8}$ & $\mathbf{8 8 . 0 9}$ \\
\hline
\end{tabular}

Table 2 reports the results obtained for the most artifact types in the test set. From table 2, it can be seen the capability of our method to detect different artifact types based on its templates preserved previously. According to this test, the overall performance was $89.438 \%$ sensitivity and $88.09 \%$ specificity when the results of all artifact types were combined. On the other hand, the total number of missed artifacts was 157 from 1180 contaminated segments specified by experts $(0.13 \%)$. These missed spikes were reexamined by neurophysiologists and classified as 47 ECG, 59 fast eye blinks and 51 chewing (EMG) artifacts. By carefully reviewing all missed artifacts, it was found that their durations ranged from 100 to $150 \mathrm{~ms}$ and amplitudes between $90-120$ $\mu \mathrm{V}$. The inability to detect these short artifacts is due to the width chosen for the initial artifact templates. Although the template length can be decreased, but the method will be more sensitive to tiny epileptic spikes resulting true EEG data distortion and unreasonable percentage of false detections (this was concluded after many experiments implemented on training data to derive the best morphological parameters of each artifact template). In total 278 false detections were verified and categorized by neurophysiologists as the following;

- 84 false-detections were Vertex spikes (quite sharply repetitive waves lasting in $200 \mathrm{~m} \mathrm{Sec}$ with amplitude up to $150-250 \mu \mathrm{V})$.

- 118 false-detections were Wicket spikes (characterized by sharp arched waveform due to temporal alpha activity).

- 76 false-detections were reported as false positives.

According to these observations, a pleasurable percentage of false detections are more satisfactory rather than detecting and removing a true epileptic spike. However, it was very important to compare our method against popular methods from the literature. In this comparison test, three very bad contaminated EEG channels (from data set 2) were acquired from $\mathrm{C} 3, \mathrm{C} 4$ and $\mathrm{Cz}$ as a reference according to the International 10-20 System. Since the most denoising methods in the literature are mainly based only on WT [29], Principle Component Analysis (PCA) [30] or ICA [31], we apply these tools to the tested data and the performance of each was compared against our presented method according to Mean Squared Error (MSE) index and the computation time consumption. Before removing artifacts, the average MSE of raw EEG data of the three EEG channels ( $\mathrm{C} 3, \mathrm{Cz}$ and $\mathrm{C} 4)$ was 93.4179. After applying the methods, average MSE was 2.18178 for our method, while 3.8911 for WT, 4.1448 for ICA and 4.9848 for PCA based method. The average running time was 0.4076 second for our method, $0.6799 \mathrm{~s}, 0.5184 \mathrm{~s}$ and $0.3876 \mathrm{~s}$ for ICA, WT and PCA based methods respectively. As the results show, in the same computing environment $(2.10 \mathrm{GHz}$, Intel Core i3 laptop), fast computational time of our algorithm shouldn't be ignored as it has the best time efficiency significantly. Moreover, the results obviously illustrate that the denoising performance of our algorithm was better than the methods based only on WT, ICA or PCA according to the Mean Squared Error (MSE) index. Although the average computation time for PCA based method was almost the same as our algorithm, its denoising performance was worse because of a lot of true EEG data loss. Therefore, considering the denoising performance and time consumption together, our method is powerful and outperforms other algorithms. Moreover, it was found in ICA based algorithm, different artifact types with various amplitudes not only disturbed the separation and artifact recognition, but also resulted in increasing the number of iterations and time computation. While our algorithm based on FastICA combined with WT (Sym8 with 5 levels) takes advantage of both ICA and WT to exploit their benefits that complement each other. So, our algorithm is powerful in noise immunity compared to other methods in the literature since FastICA in Wavelet domain has a faster convergence speed of iteration, and better realization of artifact recognition and separation. On the other side, due to the nonstationary nature of EEG signals, (meaning that characteristics as mean, variance or power-spectra, change with time [19]), determining the spectrum in time-domain, is not preferable. Since the amplitude and spectrum of EEG vary from signal to signal, subject to subject, it even varies from time to time for the same subject [32]. Also, the "rhythmic" behavior of EEG is characterized by a peak in the power spectrum at specific frequencies [33], could be used to study such changes of the signal after applying our method. Saini et al. [34] showed the efficiency of applying Lomb-Scargle transform (LST) for estimating the power spectrum, which could be convenient for our test. The LST is defined as;

For $x_{n}$, provides a vector of $\boldsymbol{n}$ observed signals $\boldsymbol{x}$ at time $\boldsymbol{n}$, the Lomb periodogram at frequency $f$ is defined by;

$$
P_{x}(f)=\frac{1}{2 \sigma^{2}}\left[\begin{array}{c}
\frac{\left[\sum_{n=1}^{N}\left(\mathrm{x}_{n}-\overline{\mathrm{x}}\right) \cos 2 \pi f\left(t_{n}-\tau\right)\right]^{2}}{\sum_{j} \cos ^{2} 2 \pi f\left(t_{n}-\tau\right)} \\
+ \\
\frac{\left[\sum_{n=1}^{N}\left(\mathrm{x}_{n}-\overline{\mathrm{x}}\right) \sin 2 \pi f\left(t_{n}-\tau\right)\right]^{2}}{\sum_{j} \sin ^{2} 2 \pi f\left(t_{n}-\tau\right)}
\end{array}\right]
$$

Where $\overline{\mathbf{x}}, \boldsymbol{\sigma}^{\mathbf{2}}$ are the mean and variance, $\boldsymbol{\tau}$ is a frequency dependent time delay defined to make the periodogram insensitive to time shift. The Lomb-Scargle Periodogram (LSP) was provided to assess the spectral performance and power evaluation of our method to study its effect on the characteristics and signal quality of the original signal after removing artifacts. To perform such test, a very simple experiment is performed using data set 3 that composed of very pure (uncontaminated) 20 EEG signals from epileptic and normal individuals as the following:-

(1) The neurophysiologists were requested to elect 20 uncontaminated (very pure) EEG signals.

(2) We contaminate these 20 pure EEG signals with white noise and different artifact types. 
(3) We apply our method to the 20 contaminated EEG signal.

(4) The LSP for average of the 20 signal was determined before and after applying our method to study the method effect on the tested signals after removing artifacts according to Lomb-Scargle Periodogram (LSP).

The following figure shows the LSP average of the 20 original uncontaminated (Fig. 5.A), contaminated (Fig. 5.B) and cleaned signals after applying our method (Fig. 5.C).

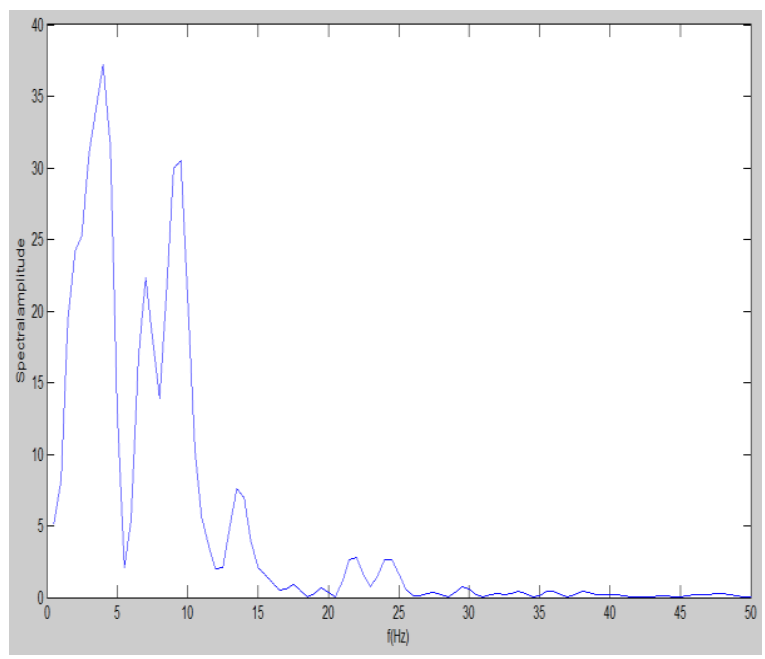

(A)

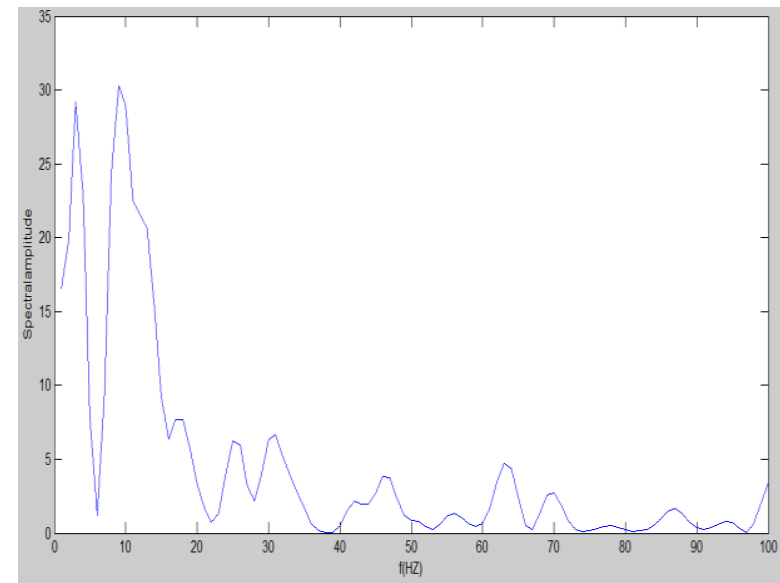

(B)

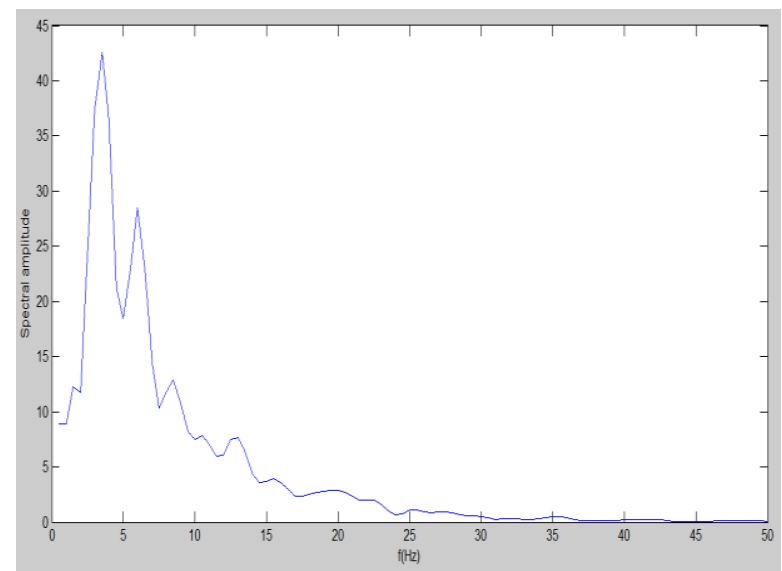

(C)

Fig 5: LSP for uncontaminated A, contaminated $B$ and cleaned signals after applying our method $\mathrm{C}$.
From Fig. 5, it can be noted that the spectrum of the denoised EEG (after applying our method) matches perfectly with the spectrum of original uncontaminated (pure) EEG signals particularly in the frequency range up to $30 \mathrm{~Hz}$. Also, one can see that the spectrum of the pure original signals (Fig. 5.A) and the denoised ones (Fig. 5.C ) are decreased to zero in the range above $30 \mathrm{~Hz}$, which is reasonable since the EEG signals do not have any useful frequency components above $30 \mathrm{~Hz}$ [35]. So, it can be understood that energy of cleaned EEG data was recovered very well, with the same distribution of power spectral density as in the pure original signals (without any tiny change or disturbance of spectral characteristics after its artifacts were removed). On the other side, the spectrum of the noised signals (Fig. 5.B) is different from the spectrum of both the pure original signals (Fig. 5.A) and the denoised ones (Fig. 5.C) in the frequency range up to and above $30 \mathrm{~Hz}$. From Fig. 5.B, the spectrum of artifact components are extended along the whole frequency range (from $0-100 \mathrm{~Hz}$ ) unlike Fig. 5.A and Fig. 5.C where there are no any spectrum elements above $30 \mathrm{~Hz}$. This important note illustrates that the different artifact types in EEG signals are spread and occupy wide frequency ranges according to the spectrum characteristics of each artifact type. The last note is crucial since it can be employed to get rid of artifacts in the frequency range above $30 \mathrm{~Hz}$ by specifying the spectral features of artifacts using power spectrum analysis tools and remove their artifact components.

\section{CONCLUSION AND FUTURE WORK}

Different artifact types are common and usually contaminate the EEG signal. Such artifacts represent a burden, embarrassment, confusion and difficulty in diagnosis and treatment of brain disorders. Thus, automatic artifacts removal attempts have received intense attention, urgently required to limit such difficulties of the traditional EEG examination. Artifacts are not just random noises and overlays disturb the true EEG morphology in the time domain but also contain underlying features in frequency and spatial domains. In this paper, we have presented a methodology based on Cosine Similarity, Wavelet Transform (sym 8) and Independent Component Analysis (FASTICA) merger to detect different 15 artifact types. In our presented method, a new application of Cosine Similarity in the field of EEG processing was used to identify any similar samples to artifact templates (preserved in the system) as a fingerprint. By analogizing their different features to that of the tested EEG windows automatically and remove the similar ones, remaining only the true uncontaminated (pure) signals. According to results, the overall performance was $89.438 \%$ sensitivity and $88.09 \%$ specificity when the results of all artifact types were combined. While we present these results that may be promising, but at the same time, it must be remembered the algorithm performance was evaluated on limited data sets. Also, the measured parameters were determined according to a visual investigation by EEG experts, utilizing the True Scan EEG system (DEYMED Diagnostic, Czech Republic), where human error during this manual inspection cannot be ignored. It is very difficult to compare our algorithm against others because the focus of this study is detecting 15 artifact types, while each technique presented in literature has its own ability to remove only one particular artifact not the most types in the same time as our method. Besides, our tested data are different from the data used to evaluate other suggested methods, with various measurements for evaluating the performance of each method, and opinions of experts about artifact patterns. From this study, we have obviously concluded that, analyzing EEG signal in a broad framework, 
including temporal, frequency, and spatial domains may be a good solution to capture different EEG artifacts by overcoming its chaotic and nonlinearity nature. On the other side, to take advantage of information and activities of adjacent channels, it is recommended that processing EEG data in a multichannel scheme can highly improve the performance rather than processing each channel individually. This important note will be taken into account in future work. Development application of our algorithm in attempts for designing an automatic seizure detection system by deriving epileptic waveform templates, identification of its similar morphology along EEG data and validation with larger data sets is our next tasks. Our presented method was tested, validated, and compared to three denoising EEG techniques on real EEG data. The comparisons between our method and other approaches based only on WT, ICA or PCA, were fulfilled to determine the performance evaluation and which artifact removal technique is the best. In fact, the results indicate that the presented method is powerful in artifact removal with fast convergence rate. For its fast computation, it provides a novel wide window for on-line preprocessing of EEG signals. Also, it can play a positive role for further research and applications in brain dysfunction treatments as deep brain and vagus nerve stimulation for seizure prevention on-line using a closed feedback loop system. So far, the performance and computational speed supported our method to be clinically applied in removing artifacts and assisting clinical neurologists in routine EEG examinations for neurological disorders diagnosis and treatment.

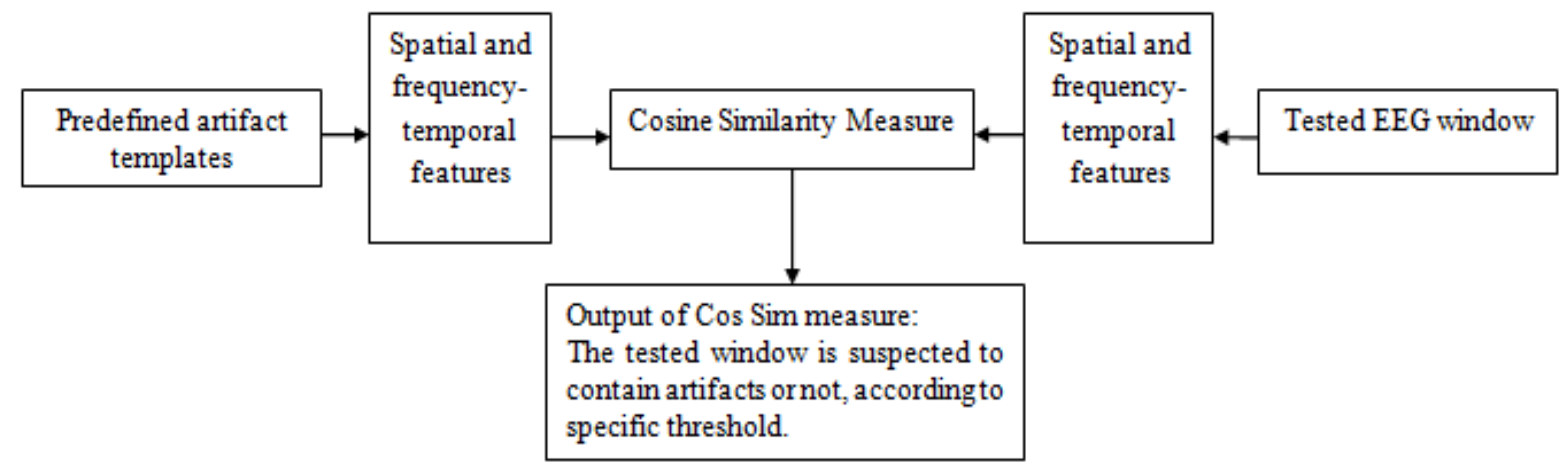

Fig 1: Features analogy of different artifact template and tested windows of raw EEG data.

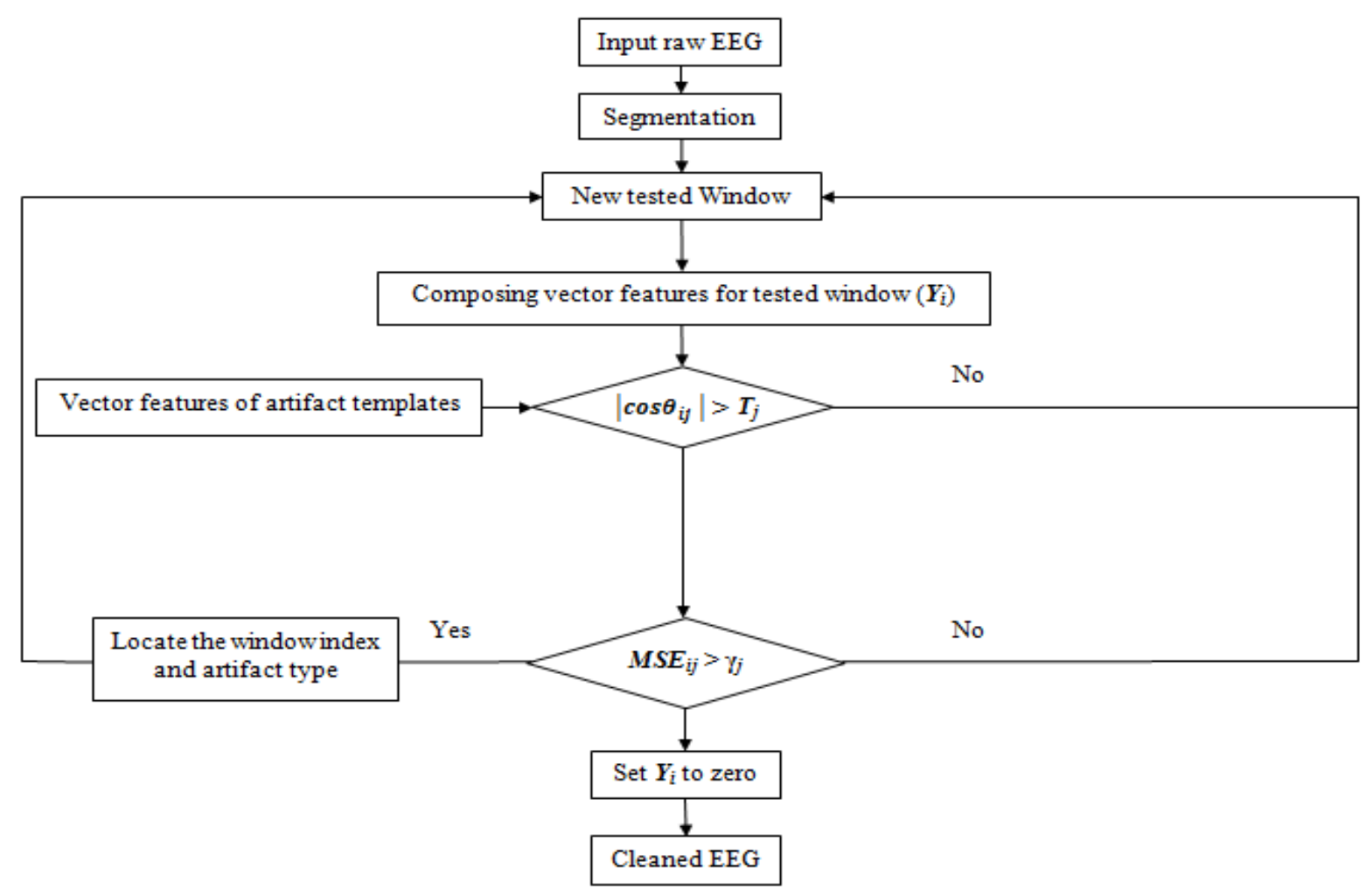

Fig 2: Schematic diagram of EEG artifacts removal 


\section{ACKNOWLEDGMENTS}

The authors gratefully appreciate the contributions of Dr. Rajeev Yadav and acknowledge his encouragement and advice.

\section{REFERENCES}

[1] Mintaze Kerem Günel "Management of Epilepsy Research, Results and Treatment" InTech, Janeza Trdine 9, 51000 Rijeka, Croatia, 2011 DOI: 10.5772/1139, available at www.intechopen.com

[2] E. Niedermeyer and F. H. Lopes da Silva, Electroencephalography: basic principles, clinical applications, and related fields, 5th ed. Philadelphia: Lippincott Williams \& Wilkins, 2010.

[3] LanlanYu, "EEG De-Noising Based on Wavelet Transformation" Bioinformatics and Biomedical Engineering, ICBBE 2009. 3rd International Conference, vol., no., pp.1-4, 11-13 June 2009.

[4] Wallstrom, Garrick L., et al. "Automatic correction of ocular artifacts in the EEG: a comparison of regressionbased and component-based methods." International journal of psychophysiology 53.2 (2004): 105-119.

[5] Correa, A. Garcés, et al. "Artifact removal from EEG signals using adaptive filters in cascade." Journal of Physics: Conference Series. Vol. 90. No. 1, IOP Publishing, 2007

[6] Jung, T-P., et al. "Removing electroencephalographic artifacts: comparison between ICA and PCA." Neural Networks for Signal Processing VIII, 1998. Proceedings of the 1998 IEEE Signal Processing Society Workshop. IEEE, 1998

[7] Jung, Tzyy-Ping, et al. "Extended ICA removes artifacts from electroencephalographic recordings." Advances in neural information processing systems (1998): 894-900.

[8] Zikov, Tatjana, et al. "A wavelet based de-noising technique for ocular artifact correction of the electroencephalogram" Engineering in Medicine and Biology, 24th Annual Conference and the Annual Fall Meeting of the Biomedical Engineering Society EMBS/BMES Conference, Proceedings of the Second Joint. Vol. 1, IEEE, 2002

[9] M. Fatourechi, A. Bashashati, R. K. Ward, and G. E. Birch, "EMG and EOG artifacts in brain computer interface systems: a survey," Clinical Neurophysiology, vol. 118, no. 3, pp. 480-494, 2007

[10] Julie Onton, Marissa Westerfield, Jeanne Townsend, Scott Makeig. "Review Imaging human EEG dynamics using independent component analysis", Neuroscience and Biobehavioral Reviews 30 (2006) 808822,Elsevier, oi:10.1016/j.neubiorev.2006.06.007

[11] Yuan Zou, John Hart and Roozbeh Jafari "Automatic EEG artifact removal based on ICA and hierarchical clustering", IEEE international conference on Acoustics, Speech and Signal Processing (ICASSP) 2012, Kyoto, Japan

[12] M. Li, Y. Cui, and J. Yang, "Automatic removal of ocular artifact from EEG with DWT and ICA Method," Applied Mathematics and Information Sciences, vol. 7, no. 2, pp. 809-816, 2013
[13] H. P. Huang, Y. H. Liu, C. P.Wang, andT. H. Huang, "Automatic artifact removal in EEG using independent component analysis and one-class classification strategy," Journal of Neuroscience and Neuroengineering, vol. 2, no. 2, pp. 73-78, 2013

[14] I. Daly, M. Billinger, R. Scherer, and G. M"uller-Putz, "On the automated removal of artifacts related to head movement from the EEG," IEEE Transactions on Neural Systems and Rehabilitation Engineering, vol. 21, no. 3, pp. 427-434, 2013

[15] Hyvärinen A. "Fast and robust fixed-point algorithms for independent component analysis", IEEE Trans Neural Netw 1999;10:626-34

[16] Frank RM, Frishkoff GA. "Automated protocol for evaluation of electromagnetic component separation (APECS): application of a framework for evaluating statistical methods of blink extraction from multichannel EEG", Clin Neurophysiol 2007; 118:8097

[17] G. Inuso, F. La Foresta, N. Mammone, and F.C Morabito "Wavelet- ICA methodology for efficient artifact removal from Electroencephalographic recordings" In Proc International Joint Conference on Neural Networks, 2011, pp. 1524-1529

[18] Janett Walters-Williams and Yan Li, “A New Approach to Denoising EEG Signals - Merger of Translation Invariant Wavelet and ICA". In International Journal of Biometric and Bioinformatics (IJBB), Volume 5, Issue 2, May 2011, pp $130-148$

[19] R. Q. Quiroga, "Quantitative analysis of EEG signals: time-frequency methods and chaos theory," Institute of Physiology-Medical University Lubeck and Institute of Signal Processing-Medical University Lubeck, 1998

[20] Lorena Orosco, Agustina Garcés Correa, Eric Laciar, (2013). "Review:A Survey of Performance and Techniques for Automatic Epilepsy Detection". Journal of Medical and Biological Engineering, 33(6): 526-537 doi: $10.5405 /$ jmbe. 1463

[21] V.V.K.D.V. Prasad, P. Siddaiah, and B. Prabhaksrs Rao, "A New Wavelet Based Method for Denoising of Biological Signals", International Journal of Computer Science and Network Security 8(1), 2008, 238-244

[22] R. Romo-Vazquez, R., Ranta, V. Louis-Dorr, and D. Maquin, "Ocular Artifacts Removal in Scalp EEG: Combining ICA and Wavelet Denoising", In the Proceedings of Physics in Signal and Image Processing (PSISP 07), 2007

[23] P. Senthil Kumar, R. Arumuganathan, K. Sivakumar, and C. Vimal "A Wavelet based Statistical Method for De-noising of Ocular Artifacts in EEG Signals" International Journal of Computer Science and Network Security (IJCSNS), 8(9), pp. 87-92, 2013

[24] Y. Song and J. Zhang, "Automatic recognition of epileptic EEG patterns via Extreme Learning Machine and multiresolution feature extraction," Expert Syst. Appl., vol. 40, no. 14, pp. 5477-5489, Oct. 2013

[25] B. Ferguson, D. Abbott, "Denoising Techniques for Terahertz Response of Biological Samples", Microelectronics Journal 32, 943-953, 2001 
[26] Ling Guo, D. Rivero, J. Dorado, Juan R.Rabunal, A. Pazos "Automatic epileptic seizure detection in EEG based on line length feature and artificial neural network" Journal of Neuroscience Methods, volume 191, issue 1, 101-109 (2010)

[27] Alain Manzo-Martínez, José A. and Camarena-Ibarrola, "A New and Efficient Alignment Technique by Cosine Distance" International Journal of Combinatorial Optimization Problems and Informatics, Vol. 4, No. 1, 2013, pp. 12-24.

[28] Mingai Li, Yan Cui, Jinfu Yang,"Automatic Removal of Ocular Artifact from EEG with DWT and ICA Method" Applied Mathematics \& Information Sciences. (2013), Appl. Math. Inf. Sci. 7, No. 2, 809-816

[29] T. Zikov, S. Bibian, G. A. Durnont, M. Huzmezan, C. R. Ries, "A wavelet based de-noising technique for ocular artifact correction of the electroencephalogram" in Proceedings of the Second Joint EMBS/BMES Conference, Houston, TX, USA, 23-26 (2009).

[30] T. P. Jung, C. Humphries, T. W. Lee "Removing Electroencephalographic Artifacts: Comparison between ICA and PCA", in Neural Networks for Signal Processing Proceedings of the 2007 IEEE Signal
Processing Society Workshop, Cambridge, England, 63 72 (2007)

[31] XIE Song-yun, ZHANG Zhen-zhong, ZHANG Weiping, and ZHAO Hai-tao, "Method and application of removing noise from EEG signals based on ICA method" Chinese Journal of Medical Imaging Technology, 23, 1562-1565 (2011)

[32] H. N. Suresh and V. Balasubramanyam "Wavelet Transforms and Neural Network Approach for Epileptical EEG”, pp. 12-17, 2012

[33] R. Yadav, R. Agarwal and M. N. S. Swamy "Detection of epileptic seizures in stereo-EEG using frequencyweighted energy", Circuits and Systems, 50th Midwest Symposium, MWSCAS 2007, pp. 77-80

[34] B. S. Saini, Dilbag Singh, Moin Uddin, and Vinod Kumar "Improved power spectrum estimation for RRinterval time series" World Acad Sci Eng Technol, (2008), 46(10):44-48

[35] P. Jahankhani, V. Kodogiannis, and K. Revett, "EEG Signal Classification Using Wavelet Feature Extraction and Neural Networks" IEEE John Vincent Atanasoff 2006 Int. Symp. Mod. Comput., pp. 120-124, Oct. 2006 